\title{
Alteraciones histopatológicas y microorganismos hallados en Rattus norvegicus infectadas con Angiostrongylus cantonensis
}

\author{
HistopathologicAl ALTERATIONS AND MICROORgANISM PRESENTS IN RATTUS NORVEGICUS \\ INFECTED WITH ANGIOSTRONGYLUS CANTONENSIS \\ Luis Solórzano-Alava ${ }^{1}$, Yolanda Narváez ${ }^{1}$, Francisco Sánchez-Amador ${ }^{1,3}$, Julio Ortiz $^{2}$
}

\section{Resumen}

\begin{abstract}
Las ratas (Rattus sp) son los hospederos definitivos del nematodo Angiostrongylus cantonensis. El objetivo de este estudio fue identificar los órganos afectados por la presencia de $A$. cantonensis (adultos) y el tipo de daño causado, así como establecer la presencia de microorganismos (bacterias y hongos) que podrían encontrarse en dichas lesiones. El estudio se realizó en 2014-2016 con 65 especímenes (60 infectadas en el laboratorio, que presentaron la larva L1 en sus deposiciones y 5 no infectadas como grupo control). Las muestras de tejido pulmonar y cardiaco fueron obtenidas en diferentes días pos-infección (dpi), teñidas con Gram y sembradas en agar sangre de caballo, agar MacConkey, medio Tioglicolato y medio Cistina Tripteína Agar (CTA). Se observaron alteraciones macro y microscópicas en tejido pulmonar y cardiaco, así como microorganismos en lesiones pulmonares tanto en el Gram como en los cultivos bacteriológicos. Se observó tejido pulmonar endurecido, células polinucleares, pus, cambios de color en el pericardio y alteraciones en el peso de los órganos. En conclusión, las ratas infectadas con Angiostrongylus cantonensis presentaron diversas alteraciones histológicas en sus órganos internos, con relación al grupo control, así como bacterias que podrían complicar el cuadro clínico.
\end{abstract}

Palabras clave: Angiostrongylus cantonensis; angiostrongilosis; bacterias; lesiones cardiacas

\section{AbStract}

Rats (Rattus sp) are the definitive hosts of the nematode Angiostrongylus cantonensis. The objective of this study was to identify the organs affected by the presence of $A$. cantonensis (adults) and the type of damage caused, as well as to establish

\footnotetext{
${ }^{1}$ Instituto Nacional Salud Pública e Investigación (INSPI), Guayaquil, Ecuador

${ }^{2}$ Clínica Veterinaria Dogtor's Cat, Quito, Ecuador

${ }^{3}$ E-mail: fisanchez@inspi.gob.ec
}

Recibido: 5 de noviembre de 2017

Aceptado para publicación: 5 de enero de 2018 


\begin{abstract}
the presence of microorganisms (bacteria and fungi) that could be found in these lesions. The study was conducted in 2014-2016 with 65 specimens ( 60 infected in the laboratory, which presented the larva L1 in their stools and 5 uninfected as a control group). Pulmonary and cardiac tissue samples were obtained on different post-infection days (dpi), stained with Gram and cultured on horse blood agar, MacConkey agar, Thioglycolate medium, Cystin medium Triptein Agar (CTA). Macro and microscopic alterations in pulmonary and cardiac tissue were observed, as well as microorganisms in lung lesions in both Gram and bacteriological cultures. Hardened lung tissue, polynuclear cells, pus, color changes in the pericardium and alterations in the weight of the organs were observed. In conclusion, the rats infected with Angiostrongylus cantonensis presented a variety of histological alterations in their internal organs, in relation to the control group, as well as bacteria that could complicate the clinical picture.
\end{abstract}

Key words: Angiostrongylus cantonensis; angiostrongyliasis; microorganisms; cardiac lesions

\section{INTRODUCCIÓN}

Angiostrongylus cantonensis fue descrito por primera vez en China afectando los pulmones de Rattus rattus y Rattus norvegicus, siendo reportado como Pulmonema cantonensis (Chen, 1935). El nematodo adulto de Angiostrongylus cantonensis vive habitualmente en las arterias pulmonares de las ratas ( $R$. rattus y $R$. norvegicus). Las larvas L1 son eliminadas con las heces y son ingeridas por un huésped intermediario, los caracoles Achatina fulica o Pomacea spp, donde se convierte en larva L2-L3, que es posteriormente ingerida por los roedores. En estos, llega al cerebro como L4L5 a los 30-33 días pos-infección, para descender al corazón y pulmones, particularmente en la arteria pulmonar donde alcanza su estado adulto (a los 45-48 días pos-infección [dpi]), cerrándose el ciclo biológico (Alicata, 1991; Martini et al., 2013).

El primer registro del parásito en Latinoamérica fue en 12 de 20 ratas (Rattus norvegicus) capturadas en seis comunidades de la provincia de La Habana, Cuba (Aguiar et al., 1981). Posteriormente fue reportado en Rattus norvegicus atrapadas en Nueva Orleans, EUU (Campbel y Little,
1988), así como en animales silvestres (Kim et al., 2002) y domésticos (Costa et al., 2000), incluyendo primates no humanos en EEUU (Duffy et al., 2004). Paralelamente, se han reportado roedores con angiostrongilosis en Puerto Rico (Andersen et al., 1986), República Dominicana (Vargas, 1992; Wang et al., 2008), Jamaica (Lindo et al., 2002), Haití (Raccurt et al., 2003), Brasil (Caldeira et al., 2007) y Ecuador (Martini et al., 2013).

En estudios previos se había encontrado que Rattus spp (ratas pardas silvestres y ratas blancas) se encontraban infectadas de manera natural con $A$. cantonensis y se había logrado reproducir el ciclo biológico en forma experimental. El presente estudio tuvo como objetivo determinar el tipo de alteraciones y lesiones anatopatológicas causadas por A. cantonensis, así como identificar microorganismos presentes en dichas lesiones.

\section{Materiales y Métodos}

El estudio se llevó a cabo en el Instituto Nacional de Salud Pública e Investigación (INSPI), Guayaquil, Ecuador. El estudio se desarrolló entre octubre de 2014 y enero de 
2016, y contó con los permisos para el uso de roedores en trabajos de investigación del Bioterio del INSPI-Veterinaria, Guayaquil; asimismo, se siguió la «Guía de manejo y cuidado de animales de laboratorio» del Ministerio de Salud de Perú (2008).

\section{Animales Experimentales}

Se utilizaron 65 ejemplares de ratas adultas albinas Rattus norvegicus, de 34 días, obtenidos en el bioterio del INSPI. De estas, 60 conformaron el grupo experimental (infectadas) y 5 fueron usadas como controles (no infectadas).

Las ratas fueron experimentalmente infectadas según el método de Morassutti et al. (2012) y Solórzano et al. (2013), vía oral, con larvas L3 de A. cantonensis (aprox. 300548) a partir de Achatina fulica que fueron previamente recolectados en La Troncal (provincia de Cañar) y en Guayaquil e inoculados en el laboratorio, siguiendo los protocolos de experimentación sobre $A$. cantonensis en roedores del Centro de Referencia Nacional de Parasitología, INSPI(Martini et al., 2013). Los cambios morfológicos que presentó $A$. cantonensis durante el ciclo de vida fueron identificados según los trabajos descriptivos de Martini et al. (2013) y Caldeira et al. (2007) y comparados con el grupo control.

Durante el experimento, se proporcionó alimentos y agua ad libitum a las ratas y se les mantuvo en un ciclo de luz/oscuridad de 12 horas, a $23-24{ }^{\circ} \mathrm{C}$ en el bioterio (Caldeira et al., 2007; Martini et al., 2013). Las ratas fueron observadas todos los días laborables de la investigación entre 08:00 y 09:00 y entre 15:00 y 16:00 horas, anotando posibles cambios de comportamiento. La presencia de $A$. cantonensis en los roedores fue corroborada mediante la eliminación de larvas L1 en las heces. Se sacrificaron 12 roedores infectados y uno del grupo control en cinco periodos: $>48,>96,>144,>170$ y $>528$ días dpi. El sacrificio se hizo por inhalación de cloroformo.
Se diseccionó la cavidad torácica, examinando el corazón, arterias pulmonares y pulmones. Se colectaron y contabilizaron los A. cantonensis adultos (Martini et al., 2013). Asimismo, los lóbulos pulmonares y los corazones fueron pesados, con y sin la presencia del parásito, en una balanza analítica MET AB54-S/FACT de $220 \mathrm{~g}$ de capacidad y 0.1 $\mathrm{mg}$ de precisión. Una vez extraídos los parásitos, se remitieron muestras de tejido pulmonar y cardiaco al Centro de Referencia Nacional de Bacteriología, INSPI-Guayaquil y el resto de los corazones al Departamento de Histo-diagnóstico Veterinario en Quito.

\section{Análisis Microbiológico}

Las muestras de tejido cardiaco y pulmonar fueron sembradas en medio de cultivo enriquecido (agar sangre de caballo), medio de cultivo específico (Agar MacConkey), medio Tioglicolato y Cistina Tripteína Agar (CTA). Se usó la tinción Gram en las cepas aisladas y fueron observadas bajo el microscopio ocular.

\section{Análisis Cito e Histopatológico}

Los corazones fueron fijados y conservados en formalina al $10 \%$, y sometidos a procedimientos de rutina para la evaluación histológica. Se tiñeron con hematoxilina eosina para su observación. Para el estudio citológico, las muestras fueron conservadas en solución salina $0.9 \%$ y teñidas con WrightDiff Quick para su observación al microscopio.

\section{Resultados}

Todos los roedores inoculados presentaron el parásito. Se recuperaron 527 nematodos adultos (machos y hembras), equivalente al $20 \%$ de larvas L3 ingeridas por los roedores. Todos los roedores infectados eliminaron larvas L1 en sus heces en los días $>48,>96,>144,>170$ y >528 dpi; así mismo, 
Cuadro 1. Pesos promedio (g) de órganos de Rattus norvegicus

\begin{tabular}{lcc}
\hline & Infectados & $\begin{array}{c}\text { No } \\
\text { infectados }\end{array}$ \\
\hline Lóbulos & $2.33 \pm 2.58$ & 1.24 \\
pulmonares & $1.07 \pm 1.38$ & 1.06 \\
\hline
\end{tabular}

todos presentaron signos clínicos de insuficiencia respiratoria (retracción intercostal, aumento de la frecuencia respiratoria) e inactividad.

El peso promedio de las 60 corazones y pulmones con parásitos A. cantonensis, fue de $20.2 \mathrm{~g}$ con un promedio de 49 nematodos por individuo. El peso promedio de los órganos se muestra en el Cuadro 1.

En la evaluación macroscópica de los ejemplares infectados (44/60 ratas $R$. norvegicus) se observó fusión de lóbulos pulmonares, los cuales presentaban tejido endurecido, punteado hemorrágico, y el tejido con coloración anaranjada a pardo oscuro. En el pericardio se encontró pequeñas zonas de tonalidad oscura. Microscópicamente se encontraron leucocitos (células polinucleares, macrófagos, eosinófilos), pus y larvas L1 de Angiostrongylus cantonensis.

En el cultivo de los tejidos pulmonares se encontró crecimiento en 4 de los 60 roedores. Así, en el día 528 dpi se encontraron cocos Gram positivos en un ejemplar que se identificaron como Staphylococcus coagulasa negativos. En el día 96 dpi se encontraron bacilos Gram negativos en otro ejemplar que se identificaron como Escherichia coli y Klebsiella pneumoniae, y en el día en el día 144 dpi en dos ejemplares se encontraron bacilos Gram positivos esporulados, posiblemente anaerobios, ya que no crecieron en las condiciones de aerobiosis. En el estudio microbiológico del tejido cardiaco no se observaron bacterias.
En el análisis histológico de los corazones con angiostrongilosis se observó epicarditis linfoplasmocítica e histiocítica, miositis intersticial linfoplasmocítica crónica y cardiomiocitos hipertróficos. Se encontraron cardiomiocitos hipertróficos compatibles con un evento de adaptación a estímulos nocivos, como el exceso de trabajo (asociado a hipertensión pulmonar por la parasitosis), que generalmente progresa a una insuficiencia cardiaca crónica. Macroscópicamente, estas lesiones se correlacionan con una discreta alteración en la relación del grosor ventricular (1:2), siendo lo normal de 1:2-1:4. Los hallazgos inflamatorios observados son compatibles con un proceso crónico e inespecífico, que en este caso pueden asociarse a la parasitosis regional.

En el análisis citológico se encontró evidencia moderada de anisocitosis y anisocariosis, hiperplasia reactiva, moderada cantidad de polimorfonucleados, neutrófilos no degenerados y eosinófilos; además de una cantidad moderada de macrófagos vacuolados y linfocitos reactivos, evidenciados por la basofilia intensa cerca de la periferia de la membrana citoplásmica, núcleos redondos, alargados o irregulares y cromatina dispuesta en forma más dispersa, tiñéndose de manera más clara que la cromatina de un linfocito en reposo.

Se evidencia un aumento marcado de la población celular miocárdica (miocitos) de morfología fusiforme de núcleos redondos a ovalados, formando agregados acinares (no compactos) evidenciando grandes espacios intercelulares en un fondo proteináceo leve eosinofilico.

Se pueden apreciar bandas o fibras musculares lisas que aparecen agrupadas $\mathrm{u}$ organizadas en capas concéntricas con inclusiones leucocitarias que indican hiperplasia reactiva. Al ser medidos, los miocitos presentan un diámetro entre 1.5 y 2.5 del tamaño del eritrocito (hipertrofia/miocitos) en vez de un diámetro de 1 a 1.5 del tamaño del eritrocito (normal). 


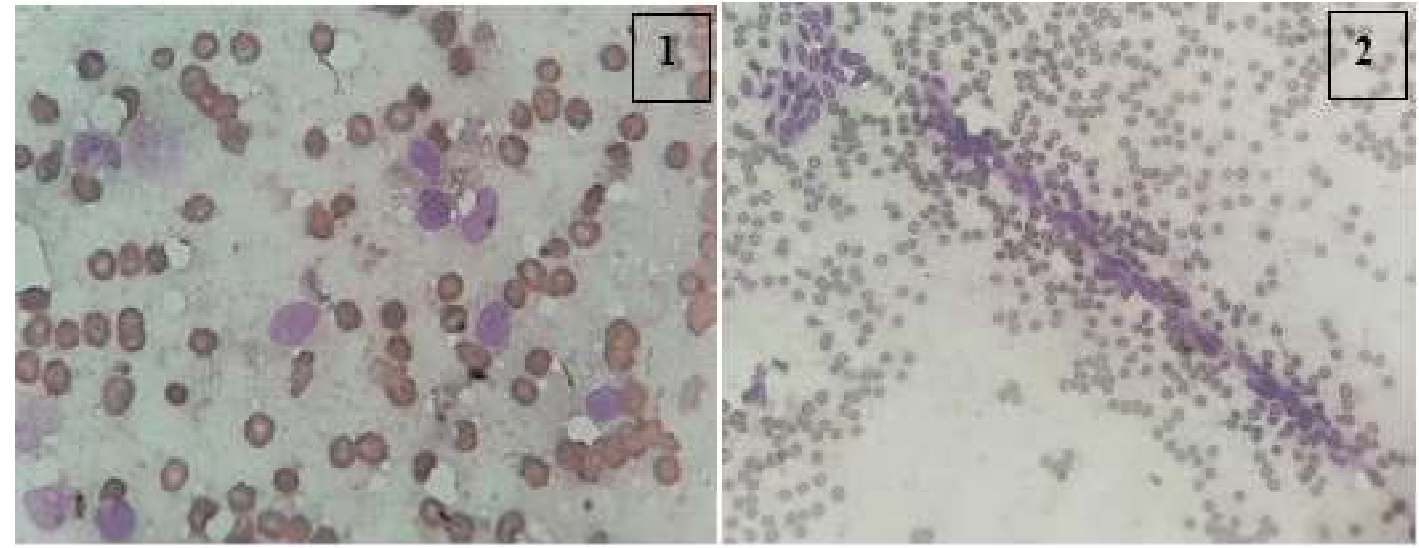

Figura 1. (1) Tejido del miocardio de Rattus norvegicus infectados con Angiostrongylus cantonensis. Se observan macrófagos vacuolados, polimorfonucleados y linfocitos reactivos. (2) Tejido del micocardio de. R. norvegicus sano con escasos macrófagos, células polimorfonucleadas y presencia de linfocitos no reactivos

En los ejemplares no infectados no se encontraron signos clínicos anormales ni presencia de microrganismos. En la evaluación microscópica se observaron escasos polimorfonucleares, neutrófilos no degenerados, escasos macrófagos y linfocitos no reactivos. Asimismo, se evidencia redes de capilares que incluyen miocitos fusiformes no displásicos (Figura 1).

Las alteraciones en el corazón y pulmones están en relación directa con el número de parásitos hallados. Además, es probable que la presencia del A. cantonensis en el corazón, arterias pulmonares y lóbulos pulmonares en $R$. norvegicus condicione un hábitat ideal para la sobreinfección bacteriana a partir de flora habitual.

\section{Discusión}

Las larvas L3 de A. cantonensis, obtenidas de huéspedes intermediarios gasterópodos, fueron ingeridas por ratas en el laboratorio. Se alcanzó a recuperar 527 parásitos a partir de 45-48 dpi; además, se constató la presencia de larvas L1 en heces. En este sentido, Morassuti et al. (2012) recuperaron $300 \mathrm{~A}$. cantonensis adultos después de 42 dpi y Kwong y Dobson (1982) recuperaron el $35 \%$ de adultos a los $90 \mathrm{dpi}$, lo cual demuestra que la técnica de ingestión oral sin sonda resulta adecuada para la recuperación de parásitos adultos. No obstante, en este estudio solo se recuperó el $20 \%$ en 45-48 dpi.

García et al. (2014) afirman que «los cambios histopatológicos en el parénquima pulmonar que demuestran los roedores con angiostrongilosis revelan la ocurrencia de necrosis y fibrosis extensa, siendo directamente relacionada con el desarrollo de hipoxia celular y cambios en las enzimas cardíacas». En el presente estudio se observó un aumento en el peso de los pulmones y en los corazones, fusión de lóbulos pulmonares, los cuales presentaban tejidos endurecidos, punteado hemorrágico, tejidos de tonos anaranjados a pardos oscuros en los cinco lóbulos pulmonares y pequeñas zonas de tonalidad oscura en el pericardio. 
En el estudio de Caldeira et al. (2007) se encontró en dos ratas sacrificadas a los 57 dpi gusanos adultos hembras en el lumen de la arteria pulmonar y los pulmones con abundante reacción granulomatosa, así como $A$. cantonensis adultos con gran número de huevos con larvogenesis. En forma similar, en este estudio se encontraron machos y hembras, tanto en el lumen de la arteria pulmonar como en los pulmones, presencia de L1 y huevos, además de alteraciones malformaciones pulmonares en roedores con $>48,>96,>144$ y $>528$ dpi.

\section{Conclusiones}

Las ratas infectadas con Angiostrongylus cantonensis presentaron alteraciones histológicas en corazón y pulmones, no presentes en el grupo control; además de signos de insuficiencia respiratoria y astenia, y presencia de bacterias que podrían complicar el cuadro clínico.

\section{Agradecimiento}

Los autores agradecen a los integrantes del Centro de Referencia Nacional de Parasitología y de Bacteriología del Instituto Nacional de Investigación en Salud Pública (INSPI), Guayaquil. Ecuador, al laboratorio de análisis y diagnóstico veterinario «ADM» y a la Clínica Veterinaria Dogtor's Cat-Quito. Asimismo, al Dr. Brayan Vásquez, Dra. Gabriela Acurio J. y Dpla. Ángela Pilar Amador Palma por la colaboración y corrección de este escrito.

\section{Literatura Citada}

1. Aguiar PH, Morera P, Pascual J. 1981. First record of Angiostrongylus cantonensis in Cuba. Am J Trop Med Hyg 30: 963-965. doi: 10.4269/ ajtmh.1981.30.963

2. Alicata JE. 1991. The discovery of Angiostrongylus cantonensis as a cause of human eosinophilic meningitis.
Parasitol Today 7: 151-53. doi: 10.1016/ 0169-4758(91)90285-V

3. Andersen E, Gubler DJ, Sorensen K, Beddard J, Ash LR. 1986. First report of Angiostrongylus cantonensis in Puerto Rico. Am J Trop Med Hyg 35: 319-322.

4. Campbell BG, Little MD. 1988. The finding of Angiostrongylus cantonensisin rats in New Orleans. Am J Trop Med Hyg 38: 568-573. doi: 10.4269/ ajtmh.1988.38.568

5. Caldeira $R$, Mendonça $C$, Oliveira $C$, Lenzi H, Graeff-Teixeira C, Lima W, Mota E, et al. 2007. First record of molluscs naturally infected with Angiostrongylus cantonensis (Chen, 1935) (Nematoda: Metastrongylidae) in Brazil. Mem Inst Oswaldo Cruz 102: 887-889. doi: 10.1590/S007402762007000700018

6. Chen TH. 1935. Un nouveau nemátode pulmonairé: Pulmonema cantonensis $\mathrm{n}$. g. n. sp., de rats de Canton. Ann Parasitol Hum Comp 13: 321-327.

7. Costa R, McClure J, Snider G, Stewart B. 2000. Verminous meningoencephalomyelitis by Angiostrongylus (Parastrongylus) cantonensis in an American miniature horse. Eq Vet Educ 12: 2-6. doi: 10.1111/j.2042-3292.2000.-tb01754.x

8. Duffy S, Miller C, Kinsella M, Lahunta A. 2004. Parastrongylus cantonesisin nonhuman primate, Florida. Emerg Infect Dis 10: 2207-2210. doi: 10.3201/ eid1012.040319

9. García JS, Dos Santos TC, Júnior AM, Tunholi VM, Tunholi VM, Mota EM, Simões R, et al. 2014. Metabolic and histopathological profile of Rattus norvegicus (Wistar) experimentally infected by Angiostrongylus cantonensis (Chen, 1935). Exp Parasitol 137: 3540. doi: 10.1016/j.exppara.2013.12.002

10. Kim D, Stewart T, Bauer W, Mitchell M. 2002. Parastrongylus (Angiostrongylus) cantonensis now endemic in Louisiana wildlife. J Parasitol 88: 10241026. doi: 10.1645/0022-3395(2002)088[1024:PACNEI]2.0.CO;2 
11. Kwong YW, Dobson C. 1982. Population dynamics of Angiostrongylus cantonensis during primary infections in rats. Parasitology 85: 399409. doi: 10.1017/S0031182000055359

12. Lindo JF, Waugh C, Hall J, Cunningham-Myrie C, Ashley D, Eberhard ML, Sullivan J, Obispo H, et al. 2002. Enzootic Angiostrongylus cantonensis in rats and snails after an outbreak of human eosinophilic meningitis, Jamaica. Emerg Infect Dis 8: 324326. doi: 10.3201/eid0803.010316

13. Martíni RL, Gómez E, Muzzio J, Solórzano L, Linda D, Amano $Y$. 2013. Caracterización morfológica de Angiostrongilus cantonensis utilizando microscopía de luz y microscopía electrónica. En: XXI Congreso Latinoamericano de Parasitología. Guayaquil: Federación Latinoamericana de Parasitología (FLAP).

14. Ministerio de Salud. 2008. Guía de manejo y cuidado de animales de laboratorio: ratón. Instituto Nacional de Salud. [Internet]. Disponible en: http:// www.ins.gob.pe/insvirtual/images/ otrpubs/pdf/guia_animales_raton.pdf
15. Morassutti A, Levert K, Pinto P, Silva A, Wilkins P, Graeff-Teixeira C. 2012. Characterization of Angiostrongylus cantonensis excretory-secretory proteins as potential diagnostic targets. Exp Parasitol 130: 26-32. doi: 10.1016/ j.exppara.2011.10.003

16. Raccurt CP. 1997. Two murine angiostrongyliasis in the Caribbean and their human consequences: a menace for Haiti? Med Trop 57: 412-413.

17. Raccurt CP, Blaise J, Durette-Desset MC. 2003. Presence of Angiostrongylus cantonensis in Haiti. Trop Med Int Health 8: 423-426.

18. Solórzano L, Martini L, Muzzio J, Hernández AH, Sarracent PJ, Rojas RL. 2013. Angiostrongylus cantonensis: un parásito emergente en Ecuador. Rev Cubana Med Trop 66: 20-33.

19. Vargas M, Gómez JD, Malek EA. 1992. First record of $A$. cantonensis (Chen, 1935). Nematoda: metastrongyloidae in the Dominican Republic. Trop Med Parasitol 43: 253-255.

20. Wang $Q$, Lai D, Zhu X, Chen X, Lun Z. 2008. Human angiostrongyliasis. Lancet Infect Dis 8: 621-630. doi: 10.1016/S1473-3099(08)70229-9 